KUNS 1088

$\mathrm{HE}(\mathrm{TH}) 91 / 13$

RIMS-778

Revised September, 2008

\title{
Discrete and Continuum Approaches to Three-Dimensional Quantum Gravity
}

\author{
Hirosi OOGURI \\ Research Institute for Mathematical Sciences \\ Kyoto University, Kyoto 606, Japan \\ and \\ NAOKI SASAKURA ${ }^{\dagger}$ \\ Department of Physics, Kyoto University \\ Kyoto 606, Japan
}

\begin{abstract}
It is shown that, in the three-dimensional lattice gravity defined by Ponzano and Regge, the space of physical states is isomorphic to the space of gauge-invariant functions on the moduli space of flat $S U(2)$ connections over a two-dimensional surface, which gives physical states in the $I S O(3)$ Chern-Simons gauge theory. To prove this, we employ the $q$-analogue of this model defined by Turaev and Viro as a regularization to sum over states. A recent work by Turaev suggests that the $q$-analogue model itself may be related to an Euclidean gravity with a cosmological constant proportional to $1 / k^{2}$, where $q=e^{2 \pi i /(k+2)}$.
\end{abstract}

\footnotetext{
* e-mail addresses : ooguri@jpnrifp.bitnet, and ooguri::kekvax

$\dagger$ e-mail address : sasakura@jpnrifp.bitnet
} 
In the two-dimensional quantum gravity, the connection between the dis-crete ${ }^{[1]}$ and the continuum ${ }^{[2,3]}$ approaches has been much explored recently ${ }^{[4,5]}$ and this facilitated our understanding on various aspects of the theory. In this letter, we point out that an example also exists in three dimensions where one can compare these two approaches.

The lattice gravity we consider here is the one originally defined by Ponzano and Regge. ${ }^{[6]}$ Consider a simplicial decomposition of a three-dimensional manifold $M$. Each three-simplex (tetrahedron) has four faces and six edges. To each edge, we assign a halfintegral number $j\left(j=0, \frac{1}{2}, 1, \ldots\right)$ and regard it as a "discretized length" of the edge. In this way, each tetrahedron is colored by an ordered set of six numbers $\left(j_{1}, \ldots, j_{6}\right)$, where boundaries of four faces of the tetrahedron are colored as $\left(j_{1}, j_{2}, j_{3}\right),\left(j_{3}, j_{4}, j_{5}\right),\left(j_{5}, j_{6}, j_{1}\right)$ and $\left(j_{6}, j_{4}, j_{2}\right)$ respectively. For the tetrahedron to be realized in the three-dimensional space, the edge-lengths $j_{i}$ 's must satisfy the triangle inequalities (i.e. $\left|j_{1}-j_{2}\right| \leq j_{3} \leq j_{1}+j_{2}$ when edges colored as $j_{1}, j_{2}$ and $j_{3}$ meet at a face of the tetrahedron). This reminds us of a decomposition rule of a tensor product of $S U(2)$ representations. Thus it is tempting to regard the edge-lengths $j_{i}$ 's as highest weights of $S U(2)$ and introduce the Racah-Wigner $6 j$-symbol

$$
\left\{\begin{array}{lll}
j_{1} & j_{2} & j_{3} \\
j_{4} & j_{5} & j_{6}
\end{array}\right\}
$$

Since the $6 j$-symbol has the tetrahedral symmetry, we can associate it to the colored tetrahedron without ambiguity.

Ponzano and Regge made a remarkable observation that, when $j$ 's are large, the $6 j$ symbol is approximated as

$$
\exp \left(-i \pi \sum_{i} j_{i}\right)\left\{\begin{array}{ccc}
j_{1} & j_{2} & j_{3} \\
j_{4} & j_{5} & j_{6}
\end{array}\right\} \sim \frac{1}{\sqrt{48 \pi V}}\left((-1)^{\sum_{i} 2 j_{i}} e^{+i\left(S_{\text {Regge }}-\pi / 4\right)}+e^{-i\left(S_{\text {Regge }}-\pi / 4\right)}\right),
$$

where $S_{\text {Regge }}$ is the Regge action ${ }^{[7]}$ for the single tetrahedron

$$
S_{\text {Regge }}=\sum_{i=1}^{6}\left(\pi-\theta_{i}\right) j_{i}
$$

with $\theta_{i}$ being the angle between outer normals of the two faces belonging to the $i$-th edge colored as $j_{i}$, and $V$ is the volume of the tetrahedron. This Regge action gives a discretized 
version of the Euclidean Einstein-Hilbert action $\int d^{3} x \sqrt{g} R$ assuming that all the faces of the tetrahedron are flat and a curvature is concentrated only on their edges. Now let us take a product of $6 j$-symbols over all the tetrahedra in $M$. Among various interference terms of $e^{+i S_{\text {Regge }}}$ and $e^{-i S_{\text {Regge }}}$, the product contains $e^{i \sum S_{\text {Regge }}}$ where the sum in the exponent is over all the tetrahedra. Thus the sum of the product of the $6 j$-symbols over all possible colorings may approximate the three-dimensional Euclidean gravity.

There are obvious problems to be overcome before taking this approach seriously. First it is not clear why one can ignore the interference terms and concentrate only on the product of the positive frequency part $e^{i S_{\text {Regge }} \star}$. Also one needs to know whether there exists a nice continuum limit of the theory. Since the $6 j$-symbol approximates the Regge action at large values of $j_{i}$ 's, the continuum limit must be such that the sum over the coloring of the tetrahedra is dominated by these $j_{i}$ 's.

Actually the sum is divergent at large $j_{i}$ 's. One might hope then, with an appropriate choice of regularization, only large values of $j_{i}$ 's become relevant in the summation and the $6 j$-symbol is well-approximated by the Regge action. The main purpose of this note is to point out that indeed there exists a regularization such that the sum over coloring of tetrahedra described in the above becomes identical to the continuum functional integral

$$
Z=\int[d e, d \omega] \exp \left(i \int e \wedge R\right)
$$

where $e$ is a dreibein, $\omega$ is a spin connection on $M$, and $R$ is a curvature two-form computed from $\omega$ as $R=d \omega+\omega \wedge \omega$. We will examine physical states in the Ponzano-Regge model and show that they can be identified with the ones in the continuum field theory (2). There is a disturbing factor of $i$ in front of the Einstein-Hilbert action $S=\int e \wedge R$ in the exponent of the integrand. We shall discuss on this issue at the end of this letter.

Now let us discuss the regularization of the sum over coloring. The method adopted by Ponzano and Regge in their original paper was simply to cut-off the sum by $j_{i} \leq L$ and

* One may also worry about the sign factor $(-1)^{\sum_{i} 2 j_{i}}$ in front of $e^{i S_{R e g g e}}$ in $(1)$. This will be taken care of if we restrict $j_{i}$ to be integral. With this restriction, most of the following discussions will go through. 
rescale it by multiplying a factor $\frac{1}{\Lambda(L)}$ per each vertex.

$$
\begin{aligned}
Z(L)= & \sum_{\substack{\text { colorings } \\
\left(j_{e} \leq L\right)}} \prod_{\text {vertices }} \frac{1}{\Lambda(L)} \prod_{\text {e:edges }}(-1)^{2 j_{e}}\left(2 j_{e}+1\right) \\
& \prod_{\text {t:tetrahedra }} \exp \left(-\pi i \sum_{i} j_{i}(t)\right)\left\{\begin{array}{lll}
j_{1}(t) & j_{2}(t) & j_{3}(t) \\
j_{4}(t) & j_{5}(t) & j_{6}(t)
\end{array}\right\} .
\end{aligned}
$$

Here $e$ and $t$ run over all the edges and the tetrahedra in $M, j_{e}$ is a coloring on $e$, and $\left(j_{1}(t), \ldots, j_{6}(t)\right)$ gives colorings on edges belonging to $t$. The rescaling factor $\Lambda(L)$ is given by

$$
\Lambda(L)=\frac{1}{2 l+1} \sum_{\substack{0 \leq i, j \leq L,|i-j| \leq l \leq i+j \\ i+j+l=0 \text { mod } \mathcal{Z}}}(2 i+1)(2 j+1) .
$$

The right-hand-side in the above does not depend on $l$ as far as $l$ is sufficiently small compared to $L$. In the limit $L \rightarrow \infty$, the factor $\Lambda(L)$ diverges as $\Lambda(L) \sim 4 L^{3} / 3$.

In the light of recent advances in quantum group technology ${ }^{[8]}$, however, it may appear more natural to regularize the summation by considering the $q$-analogue of the model. In the quantum group $U_{q}(S U(2))$ associated to $q=e^{2 \pi i /(k+2)}$ with $k$ being an integer, representations with $j \leq k / 2$ enjoy special status. Thus the cut-off of the edge-lengths $j$ will be built in as an intrinsic property in the $q$-analogue of the Ponzano-Regge model. Indeed such a model was considered by Turaev and Viro. ${ }^{[9]}$ The partition function of their model is obtained by taking the $q$-analogue of $(3)$ as ${ }^{\star}$

$$
\begin{aligned}
Z_{k}= & \sum_{\substack{\text { colorings } \\
\text { (je } \leq k / 2)}} \prod_{\text {vertices }} \frac{1}{\Lambda_{q}} \prod_{\text {e:edges }}(-1)^{2 j_{e}}\left[2 j_{e}+1\right]_{q} \\
& \prod_{\text {t:tetrahedra }} \exp \left(-\pi i \sum_{i} j_{i}(t)\right)\left\{\begin{array}{lll}
j_{1}(t) & j_{2}(t) & j_{3}(t) \\
j_{4}(t) & j_{5}(t) & j_{6}(t)
\end{array}\right\}_{q} .
\end{aligned}
$$

Here $\left\{\begin{array}{lll}j_{1} & j_{2} & j_{3} \\ j_{4} & j_{5} & j_{6}\end{array}\right\}$ is the $6 j$-symbol of the quantum group $U_{q}(S U(2))$ and $[2 j+1]_{q}$

* If the manifold $M$ has some boundaries, we multiply $\frac{1}{\sqrt{\Lambda_{q}}}$ per each vertex on the boundaries and $e^{i \pi j_{e}} \sqrt{\left[2 j_{e}+1\right]_{q}}$ per each edge in the summand. 
denotes the $q$-analogue of the integer $(2 j+1)$,

$$
[2 j+1]_{q}=\frac{q^{(2 j+1) / 2}-q^{-(2 j+1) / 2}}{q^{1 / 2}-q^{-1 / 2}} .
$$

The factor $\Lambda_{q}$ is defined by generalizing (4) as

$$
\Lambda_{q}=\frac{1}{[2 l+1]_{q}} \sum_{\substack{0 \leq i, j \leq k / 2, i+j+l \leq k \\|i-j| \leq l \leq i+j, i+j+l=0 \bmod \mathcal{Z}}}[2 i+1]_{q}[2 j+1]_{q}
$$

$\Lambda_{q}$ thus defined is independent of $l$. In fact, one can perform the summation and obtain $\Lambda_{q}=-2(k+2) /\left(q^{1 / 2}-q^{-1 / 2}\right)^{2}$.

The remarkable property of this model is that the value of $Z_{k}$ is independent of a choice of the simplicial decomposition of the three-dimensional manifold $M$. It had already been suggested by Ponzano and Regge that $Z(L \rightarrow \infty)$ is invariant under a refinement of the decomposition; one can decompose a single tetrahedron into four smaller tetrahedra by adding an extra-vertex in the middle of the original tetrahedron and $Z(\infty)$ does not change its value under this operation. Turaev and Viro have gone further and shown that $Z_{k}$ is invariant under an arbitrary Alexander transformation of simplices, which includes the refinement of a single tetrahedron as a special case. It is known that one can relate any two triangulations of $M$ by a sequence of Alexander transformations. ${ }^{[10]}$ Thus $Z_{k}$ depends only on the topology of $M$.

Although there are infinite number of Alexander transformations in three dimensions, they are represented as compositions of three local moves. The partition function $Z_{k}$ is preserved under those three moves provided

$$
\begin{gathered}
\sum_{j}(-1)^{2\left(j+j_{4}\right)}[2 j+1]_{q}\left[2 j_{4}+1\right]_{q}\left|\begin{array}{ccc}
j_{2} & j_{1} & j \\
j_{3} & j_{5} & j_{4}
\end{array}\right|_{q}\left|\begin{array}{ccc}
j_{3} & j_{1} & j_{6} \\
j_{2} & j_{5} & j
\end{array}\right|_{q}=\delta_{j_{4} j_{6}} \\
\sum_{j}(-1)^{2 j}[2 j+1]_{q}\left|\begin{array}{ccc}
j_{2} & a & j \\
j_{1} & c & b
\end{array}\right|_{q}\left|\begin{array}{ccc}
j_{3} & j & e \\
j_{1} & f & c
\end{array}\right|_{q}\left|\begin{array}{ccc}
j_{3} & j_{2} & j_{23} \\
a & e & j
\end{array}\right|_{q} \\
=\left|\begin{array}{ccc}
j_{23} & a & e \\
j_{1} & f & b
\end{array}\right|_{q}\left|\begin{array}{ccc}
j_{3} & j_{2} & j_{23} \\
b & f & c
\end{array}\right|_{q}
\end{gathered}
$$


and (6) are satisfied. Here

$$
\left|\begin{array}{lll}
j_{1} & j_{2} & j_{3} \\
j_{4} & j_{5} & j_{6}
\end{array}\right|_{q}=\exp \left(-\pi i \sum_{i} j_{i}\right)\left\{\begin{array}{lll}
j_{1} & j_{2} & j_{3} \\
j_{4} & j_{5} & j_{6}
\end{array}\right\}_{q} .
$$

These in fact are well-known formulae in conformal field theory. It has been pointed out by several groups ${ }^{[11,12]}$ that the quantum $6 j$-symbol is equal to the fusion matrix in the $S U(2)$ Wess-Zumino-Witten (WZW) model upto some phase factor. The equation (8) can then be regarded as the pentagon identity in the modular tensor category of Moore and Seiberg ${ }^{[12]}$, and $(7)$ is the unitarity relation ${ }^{\star}$.

With this connection to the WZW model, one might suspect that the lattice model of Turaev and Viro is related to the $S U(2)$ Chern-Simons (CS) gauge theory. ${ }^{[13]}$ To see if this is the case, it is useful to introduce a finite dimensional vector space $H^{(k)}(\Sigma)$, defined by Turaev and Viro, associated to a two-dimensional closed topological surface $\Sigma$. Let us fix a triangulation $t$ of $\Sigma$ and consider a vector space $C^{(k)}(\Sigma, t)$ which is freely generated by all the possible colorings of $t$ over C. Now we can define a linear map $Q_{t \rightarrow t}$ from $C^{(k)}(\Sigma, t)$ into itself as follows. Let us take a three-dimensional manifold $M$ to be of the topology $\Sigma \times[0,1] . M$ has two boundaries and both of them are isomorphic to $\Sigma$. We then fix a simplicial decomposition of $M$ in such a way that, at the boundaries of $M$, it agrees with the triangulation $t$ of $\Sigma$. With this preparation, one can compute a partition function $Z_{k}\left(c_{1}, c_{2}\right)$ as in (5), where we fix colorings $c_{1}$ and $c_{2}$ on the boundaries $\Sigma \times\{0\}$ and $\Sigma \times\{1\}$ of $M$. Now a linear map $Q_{t \rightarrow t}$ is defined as

$$
Q_{t \rightarrow t}: c \quad \rightarrow \sum_{c^{\prime} \in C^{(k)}(\Sigma, t)} Z_{k}\left(c, c^{\prime}\right) c^{\prime}
$$

Due to the invariance of $Z_{k}$ under the Alexander transformations, the definition of the map $Q_{t \rightarrow t}$ is independent of a choice of the simplicial decomposition in the interior of $M$. As a corollary of this, one can show $\left(Q_{t \rightarrow t}\right)^{2}=Q_{t \rightarrow t}$, namely $Q_{t \rightarrow t}$ is a projection operator. Thus an eigenvalue of $Q_{t \rightarrow t}$ is either 0 or 1 . We pick a subspace with an eigenvalue 1 and call it $H^{(k)}(\Sigma, t)$. In the Hamiltonian picture of the model, $Q_{t \rightarrow t}$ may be viewed as a time-evolution operator associated to the topology $\Sigma \times[0,1]$. The condition $Q_{t \rightarrow t}=1$

* Thus one could define a large class of three-dimensional lattice model of this type associated to various conformal field theories. 
should then correspond to the Hamiltonian constraint (or the Wheeler-DeWitt equation ${ }^{[14]}$ ) in the quantum gravity.

So far, we have considered $H^{(k)}(\Sigma, t)$ with respect to the fixed triangulation $t$ of $\Sigma$. One can show that two vector spaces $H^{(k)}\left(\Sigma, t_{1}\right)$ and $H^{(k)}\left(\Sigma, t_{2}\right)$ associated to different triangulations $t_{1}$ and $t_{2}$ are isomorphic if they are associated to the same topological surface $\Sigma$. To show this, we consider again the manifold $M=\Sigma \times[0,1]$, but this time $\Sigma \times\{0\}$ and $\Sigma \times\{1\}$ have different triangulations $t_{1}$ and $t_{2}$. By generalizing the construction of $Q_{t \rightarrow t}$ in the above, we can define an operator $Q_{t_{1} \rightarrow t_{2}}$ which maps $C^{(k)}\left(\Sigma, t_{1}\right)$ into $C^{(k)}\left(\Sigma, t_{2}\right)$. Since $Q_{t_{1} \rightarrow t_{2}} \circ Q_{t_{2} \rightarrow t_{1}}$ is equal to the map $Q_{t_{1} \rightarrow t_{1}}$ which acts as an identity operator on $H^{(k)}\left(\Sigma, t_{1}\right)$, the restriction of $Q_{t_{1} \rightarrow t_{2}}$ on $H^{(k)}\left(\Sigma, t_{1}\right)$ defines an isomorphism between $H^{(k)}\left(\Sigma, t_{1}\right)$ and $H^{(k)}\left(\Sigma, t_{2}\right)$. Thus we may drop $t$ in $H^{(k)}(\Sigma, t)$ and call it a space of "physical states" in the Turaev-Viro model.

Now we are in a position to establish a connection between the lattice model of Ponzano and Regge and the continuum field theory given by (2). First let us show that the space of physical states in the Ponzano-Regge model $H^{(k=\infty)}(\Sigma)$ is isomorphic to $\mathcal{F}($ flat), the space of gauge-invariant functions over the moduli space of flat $S U(2)$ connections. To study the structure of $\mathcal{F}$ (flat), it is easier to start with $\mathcal{F}$ consisting of gauge-invariant functions on the space of all $S U(2)$ connections. An element of $\mathcal{F}$ is constructed from Wilson-line operators $U_{j}(x, y)\left(x, y \in \Sigma, j=0, \frac{1}{2}, 1, \ldots\right)$,

$$
U_{j}(x, y)=P \exp \left(\int_{x}^{y} A^{a} t_{j}^{a}\right),
$$

where $P \exp$ denotes the path ordered exponential and $t_{j}^{a}(a=1,2,3)$ is the spin- $j$ generator of $S U(2)$. Under a gauge transformation, $A \rightarrow \Omega^{-1} A \Omega+\Omega^{-1} d \Omega$, the Wilson-line operator behaves as $U(x, y) \rightarrow \Omega(x)^{-1} U(x, y) \Omega(y)$. Now consider their tensor product $\otimes_{i} U_{j_{i}}\left(x_{i}, y_{i}\right)$. To make this gauge-invariant, we need to contract group indices of $U_{i}$ 's so that the gauge factor $\Omega$ cancels out. Invariant tensors we can use to contract the indices are the ClebschGordan (CG) coefficient $\left\langle j_{1} j_{2} m_{1} m_{2} \mid j_{3} m_{3}\right\rangle$ and the metric $g_{m m^{\prime}}^{(j)}=\sqrt{2 j+1}\left\langle j j m m^{\prime} \mid 0,0\right\rangle=$ $(-1)^{j-m} \delta_{m+m^{\prime}, 0}$. The gauge-invariant function constructed this way corresponds to a colored trivalent graph $Y$ on $\Sigma$, where a contour from $x$ to $y$ in $Y$ corresponds to a 
Wilson-line $U(x, y)$ and three-point vertices in $Y$ represent the CG-coefficients ${ }^{\star}$. Thus, to each colored trivalent graph $Y$, we can associate a function $\Psi_{Y} \in \mathcal{F}$. In general, a gauge-invariant function of $A$ is a linear combination of such $\Psi_{Y}$ 's. Thus $\mathcal{F}$ is isomorphic to a vector space $\tilde{C}(\Sigma)$ which is freely generated by colored trivalent graphs on $\Sigma$. The isomorphism is defined as $\sum_{i} a_{i} Y_{i} \in \tilde{C}(\Sigma) \rightarrow \sum_{i} a_{i} \Psi_{Y_{i}} \in \mathcal{F}$, where $Y_{i}^{\prime}$ 's are colored trivalent graphs.

The function $\Psi_{Y}(A)$ can be regarded as an element of $\mathcal{F}($ flat $)$ by simply restricting its domain to the space of flat connections. The map $Y \rightarrow \Psi_{Y} \in \mathcal{F}$ (flat) however, is not injective (i.e. two different graphs $Y$ and $Y^{\prime}$ may give the same function $\Psi_{Y}=\Psi_{Y^{\prime}}$ upon the restriction). In general, if two graphs $Y$ and $Y^{\prime}$ are homotopic, the corresponding functions $\Psi_{Y}$ and $\Psi_{Y^{\prime}}$ have the same value on flat connections. To each colored trivalent graph $Y$, one can associate a colored triangulation as a dual graph. Especially if two graphs $Y$ and $Y^{\prime}$ are homotopically inequivalent, they correspond to distinct colored triangulations. The isomorphism between $\tilde{C}(\Sigma)$ and $\mathcal{F}$ then induces a homomorphism $\varphi: C(\Sigma) \rightarrow \mathcal{F}($ flat $)$, where $C(\Sigma)=\oplus_{t} C^{(k=\infty)}(\Sigma, t)$ is a vector space freely generated by colored triangulations. Thus in order to study the structure of $\mathcal{F}$ (flat), we would like to identify the kernel of $\varphi$.

Since $\Psi_{Y}$ on a flat connection is invariant under homotopy move of $Y$, we may pick any open Wilson-line in $Y$ and make its length to be arbitrary small without changing the value of $\Psi_{Y}$. The corresponding Wilson operator can then be replaced by an identity, and group indices of CG-coefficients at two end-points of the Wilson-line are simply summed over. Now there is a formula which relates two different ways of summing CG-coefficients ${ }^{[15]}$,

$$
\begin{gathered}
\sum_{m_{6}}\left\langle j_{2} j_{4} m_{2} m_{4} \mid j_{6} m_{6}\right\rangle\left\langle j_{1} j_{6} m_{1} m_{6} \mid j_{5} m_{5}\right\rangle \\
=\sum_{j_{3}} e^{\pi i\left(j_{3}+j_{6}\right)} \sqrt{\left(2 j_{3}+1\right)\left(2 j_{6}+1\right)} e^{-\pi i \sum_{i} j_{i}}\left\{\begin{array}{ccc}
j_{1} & j_{2} & j_{3} \\
j_{4} & j_{5} & j_{6}
\end{array}\right\} \\
\sum_{m_{3}}\left\langle j_{1} j_{2} m_{1} m_{2} \mid j_{3} m_{3}\right\rangle\left\langle j_{3} j_{4} m_{3} m_{4} \mid j_{5} m_{5}\right\rangle .
\end{gathered}
$$

* Subtlety arises when there are two Wilson-lines intersecting with each other. In such a case, we cut the Wilson-lines at the intersecting point and use the identity $g_{m_{1} n_{1}}^{\left(j_{1}\right)} g_{m_{2} n_{2}}^{\left(j_{2}\right)}=\sum_{j, m, n} g_{m n}^{(j)}\left\langle j_{1} j_{2} m_{1} m_{2} \mid j m\right\rangle\left\langle j_{1} j_{2} n_{1} n_{2} \mid j n\right\rangle$ to replace the intersection by two vertices and an infinitesimal Wilson-line connecting them. 
We can use this to obtain the following relation.

$$
\Psi_{\tilde{Y}_{j_{6}}}=\sum_{j_{3}} e^{\pi i\left(j_{3}+j_{6}\right)} \sqrt{\left(2 j_{3}+1\right)\left(2 j_{6}+1\right)} e^{-\pi i \sum_{i} j_{i}}\left\{\begin{array}{ccc}
j_{1} & j_{2} & j_{3} \\
j_{4} & j_{5} & j_{6}
\end{array}\right\} \Psi_{Y_{j_{3}}},
$$

where the graph $Y_{j_{3}}$ contains a Wilson-line of spin- $j_{3}$ connecting lines with $j_{1}$ and $j_{2}$ to lines with $j_{4}$ and $j_{5}$ at the two end-points, while $\tilde{Y}_{j_{6}}$ is obtained by replacing this Wilson-line in $Y_{j_{3}}$ by its dual line of spin- $j_{6}$ connecting $j_{1}$ and $j_{5}$ to $j_{2}$ and $j_{4}$.

If a graph contains a contractible loop with several external lines, by repeatedly using (9), the loop can be recombined into a tree with a one-loop tadpole. The tadpole can be made arbitrarily small, and the infinitesimal tadpole can be removed by using

$$
\sum_{m m^{\prime}} g_{m m^{\prime}}^{(j)}\left\langle j j m m^{\prime} \mid J M\right\rangle=\sqrt{2 j+1} \delta_{J, 0} \delta_{M, 0}
$$

For example, if $Y$ contains a loop with three external-lines $j_{1}, j_{2}$ and $j_{3}$ attached, we can shrink the loop to obtain another graph $Y^{\prime}$ where the three lines meet at one point. The corresponding functions $\Psi_{Y}$ and $\Psi_{Y^{\prime}}$ are related as

$$
\Psi_{Y}=\sqrt{\left(2 l_{12}+1\right)\left(2 l_{23}+1\right)\left(2 l_{31}+1\right)} e^{-\pi i \sum_{i=1}^{3} j_{i}}\left\{\begin{array}{lll}
j_{1} & j_{2} & j_{3} \\
l_{23} & l_{31} & l_{12}
\end{array}\right\} \Psi_{Y^{\prime}}
$$

where $l_{i j}$ is the color of the segment of the loop in $Y$ connecting $j_{i}$ and $j_{j}$. Using a variation of the analysis in Appendix D of [16], one can show that all other relations among $\Psi_{Y}$ 's in $\mathcal{F}$ (flat) are generated from (10) and (12).

From (10) and (12), one sees that if $c \in C^{(k=\infty)}(\Sigma, t)$ and $c^{\prime} \in C^{(k=\infty)}\left(\Sigma, t^{\prime}\right)$ are related as $c^{\prime}=Q_{t \rightarrow t^{\prime}} c$, they are mapped into the same function in $\mathcal{F}$ (flat) by $\varphi$. For example, (12) is realized as a process of attaching a tetrahedron on a single triangle in $t$, while (10) is an operation to recombine two neighboring triangles on $\Sigma$ into ones in a dual position. Since the map $Q_{t \rightarrow t^{\prime}}$ can be constructed from these two moves, the kernel of the homomorphism $\varphi$ is characterized by the relations given by $Q_{t \rightarrow t^{\prime}}$ on $C(\Sigma)$, namely $C(\Sigma) / \operatorname{ker}(\varphi) \simeq H^{(k=\infty)}(\Sigma)$. By the homomorphism theorem, we obtain $\mathcal{F}($ flat $) \simeq$ $H^{(k=\infty)}(\Sigma)$. 
Now that we have found $H^{(k=\infty)}(\Sigma) \simeq \mathcal{F}$ (flat), we would like to connect it to the physical Hilbert space of the continuum field theory (2). Following the observation by Witten $^{[17]}$ in the case of the Lorentzian gravity, we regard $\int e \wedge R$ as the CS-action whose gauge group is $I S O(3)$. In the Hamiltonian formulation of the $I S O(3)$ CS-theory, the timelike components of $e$ and $\omega$ do not have their canonical conjugate variables, while the spacelike components $e_{i}$ and $\omega_{i}(i=1,2)$ are conjugate to each other. The variable $e_{0}$ imposes a constraint that the $S U(2)$ connection $\omega_{i}$ on $\Sigma$ is flat ${ }^{\star}$, and the physical subspace is the cotangent space of the moduli space $\mathcal{M}(\Sigma)$ of flat $S U(2)$ connections over $\Sigma$. Thus a physical wave function in the $I S O(3)$ CS-theory can be regarded as a gauge-invariant function on $\mathcal{M}(\Sigma)$. This establishes the connection between the Ponzano-Regge lattice model and the continuum field theory (2).

What about the $q$-analogue model? Since the quantum $6 j$-symbol gives the fusion matrix of the WZW model, it is natural to expect that $H^{(k)}$ is related to the space $\mathcal{H}^{(k)}$ of conformal blocks of the WZW model. When $k=1$, the structure of $H^{(k=1)}(\Sigma)$ is rather simple. In this case, $U_{q}(S U(2))$ has two representations $j=0$ or $1 / 2$, and an element of $C^{(k=1)}(\Sigma, t)$ may be viewed as a collection of closed monochromatic cycles on $\Sigma$. The constraint $Q_{t \rightarrow t}=1$ then implies that $H^{(k=1)}(\Sigma)$ is the space of functions on $H_{1}\left(\Sigma, \mathcal{Z}_{2}\right)$, and it is isomorphic to $\mathcal{H}^{(k=1)} \otimes \overline{\mathcal{H}^{(k=1)}}$. For $k \geq 2$, we must deal with a graph with $k$ different colors, $j=1 / 2,1, \ldots, k / 2$. However, if a contour in the graph is colored by $j \geq 1$, by using a map $Q_{t \rightarrow t^{\prime}}$, it can be decomposed into a network consisting only of contours with $j=1 / 2$. We have examined the structure of $H^{(k)}(\Sigma)$ for lower genus $\Sigma$ and found that they are isomorphic to $\mathcal{H}^{(k)} \otimes \overline{\mathcal{H}^{(k)}}$. Detailed of this procedure and its extension to higher genera will be discussed elsewhere.

This suggests that the partition function $Z_{k}$ of the Turaev-Viro model is equal to the absolute-value-square of the partition function of the $S U(2)$ CS-theory when $M$ is orientable. Recently we were informed that this had indeed been proven by Turaev in a rather different approach ${ }^{\star}$. The partition function $Z_{k}$ of the $q$-analogue lattice model

* As we mentioned before, we may either include half-integral $j$ 's in the summation in (3) and (4) or restrict $j$ 's to be integral. In the latter case, $\omega_{i}$ should be viewed as an $S O(3)$ connection rather than $S U(2)$.

* We thank T.Kohno for informing this to us and T.Takata for sending us a copy of a hand-written manuscript [18] by Turaev. 
should then be expressed as

$$
\begin{aligned}
Z_{k}=\left|\int[d A] \exp \left(i \frac{k}{4 \pi} \int\left(A d A+\frac{2}{3} A^{3}\right)\right)\right|^{2} \\
=\int[d A, d B] \exp \left(i \frac{k}{4 \pi} \int\left(A d A+\frac{2}{3} A^{3}\right)-\left(B d B+\frac{2}{3} B^{3}\right)\right) \\
=\int[d e, d \omega] \exp \left(i \int e \wedge R+\frac{\lambda_{k}}{3} e \wedge e \wedge e\right) \\
\omega=\frac{1}{2}(A+B), \quad e=\frac{k}{8 \pi}(A-B),
\end{aligned}
$$

where the "cosmological constant" $\lambda_{k}$ is equal to $(4 \pi / k)^{2}$. Due to the cosmological term, a classical solution to $R+\lambda_{k} e \wedge e=0$ would be a three-dimensional sphere of radius $k / 4 \pi$. It is intriguing note that the maximum length $k / 2$ of a geodesic on the sphere coincides with the maximum value of $j$ for $U_{q}(S U(2))$. In the limit of $k \rightarrow \infty$, the cosmological constant vanishes and the above equation reduces to $(2)$.

To regard (2) or (13) as an Euclidean functional integral for quantum gravity, the factor $i$ in front of the action is disturbing. One might try to eliminate it by rotating the contour of the $e$-integral, but the resulting functional integral would then be divergent. The problem is that the sign of $\int e \wedge e \wedge e$ is indefinite unlike $\int \sqrt{g}$, which is clearly positive definite, and one does not know how to define an Euclidean functional integral in the first order formalism of $e$ and $\omega$. The volume form could be made positive definite by integrating over $e$ with fixed orientation only ( $e \rightarrow-e$ flips an orientation in three dimensions), but it is not clear whether the resulting theory is renormalizable.

This issue would be addressed by studying a Lorentzian version of the lattice model based on infinite dimensional unitary representations of $S O(2,1)$. A work in this direction is now in progress.

It is straightforward, at least in the limit of $k \rightarrow \infty$, to extend the above analysis to another compact Lie group $G$. In general there are more than one way to contract Wilson-line operators at a vertex. This means that, in such a lattice model, we put colors on faces of tetrahedra in $M$ as well as on edges. This model should be equivalent to what is called the BF-theory ${ }^{[19] \star}$ for the group $G$.

\footnotetext{
* In three dimensions, the $I S O(3)$ CS-theory may be regarded as the $S U(2)$ BF-theory.
} 


\section{Acknowledgments}

The authors would like to thank R.Sorkin for discussion on the model of Ponzano and Regge, and A.Shapere for bringing the paper by Turaev and Viro into their attention. They are grateful to T.Kohno and T.Takata for information on the work by Turaev.

\section{REFERENCES}

1. J. Ambjørn, B. Durhuus and J. Frölich, Nucl. Phys. B257 [FS14] (1985) 433;

F. David, Nucl. Phys. B257 [FS14] (1985) 45;

V.A. Kazakov, I.K. Kostov and A.A. Migdal, Phys. Lett. 157B (1985) 295.

2. V.G. Knizhnik, A.M. Polyakov and A.B. Zamolodchikov, Mod. Phys. Lett. A3 (1988) 819.

3. F. David, Mod. Phys. Lett. A3 (1988) 1651;

J. Distler and H. Kawai, Nucl. Phys. B321 (1989) 509.

4. V.A. Kazakov, Mod. Phys. Lett. A4 (1989) 2125.

5. M.R. Douglas and S.H. Shenker, Nucl. Phys. B335 (1990) 635;

D.J. Gross and A.A. Migdal, Phys. Rev. Lett. 64 (1990) 127;

E. Brezin and V.A. Kazakov, Phys. Lett. 236B (1990) 144.

6. G. Ponzano and T. Regge, in Spectroscopic and Group Theoretical Methods in Physics, ed. F. Bloch (North-Holland, Amsterdam, 1968).

7. T. Regge, Nuovo Cimento 19 (1961) 558.

8. M. Jimbo, Lett. Math. Phys. 10 (1985) 63;

V.G. Drinfeld, "Quantum Groups," in Proc. Intl. Congress of Mathematicians, Berkeley, California, (1986) p.798.

9. V.G. Turaev and O.Y. Viro, "State Sum Invariants of 3-Manifolds and Quantum 6j-Symbols," preprint (1990).

10. J.W. Alexander, Ann. Math. 31 (1930) 292.

11. A. Tsuchiya and Y. Kanie, in Conformal Field Theory and Solvable Lattice Models, Advanced Studies in Pure Mathematics, 16 (1988) 297;

T. Kohno, in "Quantized Universal Enveloping Algebras and Monodromy of Braid 
Groups," Nagoya preprint (1988);

L. Alvarez-Gaumé, C. Gomez and G. Sierra, Phys. Lett. 220B (1989) 142;

G. Moore and N. Reshetikhin, Nucl. Phys. B328 (1989) 557.

12. G. Moore and N. Seiberg, Phys. Lett. 212B (1988) 451;

Commun. Math. Phys. 123 (1989) 77.

13. E. Witten, Commun. Math. Phys. 121 (1989) 351.

14. B.S. DeWitt, Phys. Rev. 160 (1967) 1113;

J.A. Wheeler, in Batelle Recontres, eds. C.M. DeWitt and J.A. Wheeler (W.A. Benjamin, inc., New York, 1968).

15. See for example, D.A. Varshalovich, A.N. Moskalev and V.K. Khersonskii, Quantum Theory of Angular Momentum (World Scientific, Singapore, 1988).

16. B.V. Boulatov, V.A. Kazakov, I.K. Kostov and A.A. Migdal, Nucl. Phys. B275 [FS17] (1986) 641.

17. E. Witten, Nucl. Phys. B311 (1988/89) 46.

18. V.G. Turaev, "Quantum Invariants of 3-Manifolds and a Glimpse of Shadow Topology," hand-written manuscript (1991).

19. I. Oda and S. Yahikozawa, preprint IC-90-44 (1990). 